\title{
Age-Related Changes in Prefrontal and Hippocampal Contributions to Relational Encoding
}

\author{
Donna Rose Addis ${ }^{1}$, Kelly S. Giovanello², Mai-Anh Vu ${ }^{3}$, and Daniel L. Schacter ${ }^{3}$ \\ ${ }^{1}$ School of Psychology and the Centre for Brain Research, The University of Auckland, \\ NewZealand ${ }^{2}$ Department of Psychology and Biomedical Research Imaging Center, The \\ University of North Carolina, Chapel Hill, N.C. ${ }^{3}$ Department of Psychology, Harvard University, \\ Cambridge, M.A.
}

\begin{abstract}
Age-related declines in relational encoding are well documented. It remains unclear, however, whether such declines reflect dysfunction of (1) ventrolateral prefrontal cortex (VLPFC) and deficient generation of associations; and/or (2) hippocampal dysfunction and impoverished binding of associations. In order to separate VLPFC and hippocampal contributions to relational encoding, we manipulated the generative demands of the encoding task by varying the number of semantic associations between the to-be-encoded information (three words). Thus, trials with fewer semantic associations (lower-association trials) require more generative processing during encoding, relative to trials in which more semantic associations are provided for binding (higherassociation trials). Parametric modulation analyses on successfully encoded items revealed that, unlike younger adults, older adults did not show an up-regulation of VLPFC activity during lowerassociation trials. In contrast, hippocampal activity in both older and younger adults was greater in higher- relative to lower-association trials. Moreover, recognition accuracy improved significantly in both groups with the provision of more semantic associations, indicating the both younger and older adults benefitted from this form of encoding support. Our findings suggest that left VLPFC dysfunction may underlie relational encoding deficits in older adults, but that when provided with associations to bind, hippocampal activity in older adults is comparable to young, consistent with their increased recognition accuracy under conditions of encoding support.
\end{abstract}

\section{Keywords}

aging; associative encoding; episodic; fMRI; parametric modulation

\section{Introduction}

Relational encoding, the ability to associate or integrate unrelated pieces of information, depends upon two distinct mnemonic mechanisms: Generation of an association between the to-be-encoded items and the integration or binding of those items into a cohesive memory

\footnotetext{
(C) 2013 Elsevier Inc.

Corresponding author: Donna Rose Addis, School of Psychology, The University of Auckland, Private Bag 92019, Auckland, New Zealand, Tel: +64 (9) 3737599 extn 88552; Fax: +64 (9) 373 7450, d.addis@ auckland.ac.nz.

Publisher's Disclaimer: This is a PDF file of an unedited manuscript that has been accepted for publication. As a service to our customers we are providing this early version of the manuscript. The manuscript will undergo copyediting, typesetting, and review of the resulting proof before it is published in its final citable form. Please note that during the production process errors may be discovered which could affect the content, and all legal disclaimersthat apply to the journal pertain.
} 
trace (Addis and McAndrews, 2006; Fernandez and Tendolkar, 2001). Prior studies in younger adults suggest that controlled, strategic processes such as the generation of associations to organize items are mediated by the prefrontal cortex (PFC; Buckner, 2003). A meta-analysis of 26 neuroimaging studies of verbal and visual encoding reported that the left ventrolateral PFC (VLPFC) and dorsolateral PFC were the most reliably activated regions (Spaniol et al., 2009). Moreover, it appears that left VLPFC is particularly engaged during relational relative to item encoding (e.g., Achim and Lepage, 2005; Addis and McAndrews, 2006; Fletcher et al., 2000; Lepage et al., 2000; Mottaghy et al., 1999; Murray and Ranganath, 2007; Prince et al., 2005), especially when the task necessitates the generation of semantic associations linking the to-be-encoded items (Achim and Lepage, 2005; Addis and McAndrews, 2006; Fletcher et al., 2000; Lepage et al., 2000). Once organized, to-be-encoded information must be linked together to form an integrated memory trace, a process subserved by the medial temporal lobe (MTL), particularly the hippocampus (Eichenbaum et al., 2007; Moscovitch, 1992). Indeed, neuroimaging studies of relational encoding consistently report hippocampal activity (Chua et al., 2007; Davachi et al., 2003; Henke et al., 1999; Jackson and Schacter, 2004; Kirwan and Stark, 2004; Prince et al., 2005; Staresina and Davachi, 2008, 2009).

It is well established that older adults experience declines in relational memory (for a review, see Old and Naveh-Benjamin, 2008), which may arise from deficient prefrontallymediated strategic processes (Anderson and Craik, 2000; Craik, 1986; Davis et al., 2013; Jennings and Jacoby, 1993; Light et al., 2000; Moscovitch and Winocur, 1995) and/or impaired hippocampal-dependent binding mechanisms (Burke and Light, 1981; Chalfonte and Johnson, 1996; Lyle et al., 2006; Mitchell et al., 2000; Naveh-Benjamin, 2000; Ryan et al., 2007). While some studies report no age-related differences in either VLPFC or hippocampal activity during encoding (e.g., Leshikar et al., 2010; Morcom et al., 2003), other studies have demonstrated age-related decreases in activity in these regions during successful relational encoding. Age-related reductions in hippocampal activity are consistently reported during relational encoding of face-name (Sperling et al., 2003), facescene (Dennis et al., 2007) and pairs of object arrays (Mitchell et al., 2000). Cabeza and colleagues (1997) observed age-related decreases in left VLPFC activity during intentional learning of word pairs and suggested a direct relationship between VLPFC function and older adults' deficits in forming new semantic associations, particularly given the role of this region in semantic processing (Cabeza and Nyberg, 2000).

However, the presence of age-related decreases in neural activity, particularly in VLPFC (Cabeza et al., 1997), may be related to whether or not encoding is actually successful (cf. Leshikar et al., 2010; Morcom et al., 2003); unsuccessful encoding attempts are likely associated with less neural activity in these key regions. Moreover, because the ability to strategically generate associations between to-be-encoded information and the binding of these associations are inherently related processes during successful encoding, it may be that the demands on strategy use are an important factor in determining whether or not neural activity during successful encoding is decreased in older adults. For instance, the apparent decline in hippocampal activity evident in many studies could reflect an inability to bind associations. Alternatively, it could it be indirectly related to PFC dysfunction and the failure to generate associations in the first place, thereby meaning there are no associations to bind. The latter explanation would suggest that age-related declines in VLPFC and MTL regions should co-occur, and while some studies only report age-related decreases in the MTL (Daselaar et al., 2003a, b), other studies report them for both the VLPFC and MTL (Dennis et al., 2008a; Grady et al., 1995; Sperling et al., 2003). Although these latter studies are therefore consistent with the notion that dysfunction in both prefrontal and hippocampal regions contributes to relational memory deficits in older adults, understanding how activity 
increases and decreases in these regions according to the strategic demands of encoding is an important task.

One approach to examining this question is to train older adults in the use of semantic encoding strategies (Kirchhoff et al., 2012). While a recent study has demonstrated that strategy training increased activity in prefrontal regions, such as VLPFC, there were no reported changes in hippocampal function (Kirchoff et al., 2012). Another approach is to vary the generative demands of the task through the provision of encoding support - such as presenting associations between to-be-encoded items. Behavioral studies have demonstrated that providing older adults with encoding support can ameliorate or eliminate apparent agerelated deficits in encoding (e.g., Bunce, 2003; McGillivray and Castel, 2010) and diminish age-related differences in left VLPFC (Logan et al., 2002). Because generative difficulties may result in having fewer associations to bind (cf. Daselaar et al., 2003b, for a similar view), explicitly providing the associations between to-be-encoded items may reduce apparent age-related differences in hippocampal activity. One recent study has examined whether age-related differences in neural activity differed according to the relatedness of word-pairs (Leshikar et al., 2010). Although no age-related differences in MTL activity were observed, it is notable that hippocampal activity was not modulated by relatedness in either age-group.

In order to examine whether changing the strategic demands of a relational encoding task influences age-related differences in VLPFC and hippocampal activity, we used a semantic relatedness paradigm in which the generative demands of encoding could be varied. Trials comprised three words (triads consisting of one category and two exemplar words), and we varied the number of semantic associations between the words. For instance, in it might be that both, one or none of the exemplar words are exemplars of the named category (see Figure 1). Thus, trials on which there are no semantic associations between the words (lower-association trials) require the generation of more associations during encoding. In contrast, in trials on which all words are semantically associated (higher-association trials), more associations are provided for binding. Previous studies using this paradigm indicate that in younger adults, activity during successful encoding varies parametrically depending on the generative demands of the trial: Encoding of lower-association trials are associated with an up-regulation of activity in VLPFC relative to higher-association trials; conversely, higher-relative to lower-association trials are associated with increased hippocampal activity (Addis and McAndrews, 2006; Lepage et al., 2000).

With respect to aging, we hypothesized that if age-related deficits in relational encoding reflect deficient generation of associations, older adults will exhibit less up-regulation of left VLPFC than younger adults when the strategic demands of the task are greater (i.e., on lower-association trials), even when controlling for encoding success. Younger adults, on the other hand, should be able to modulate VLPFC activity according to the generative demands of the encoding task (i.e., lower- to higher-association trials). However, if encoding deficits reflect hippocampal dysfunction and impoverished binding of associations, then relative to younger adults, hippocampal activity and encoding performance in older adults will not increase even when associations are explicitly provided on higher-association trials.

\section{Material and Methods}

\subsection{Participants}

All participants gave informed written consent in a manner approved by the Harvard and Massachusetts General Hospital Institutional Review Boards. All subjects were righthanded, fluent in English, and had no history of neurological or psychiatric disorders. 
2.1.1 Older adults-Eighteen older adults were recruited for this study. Two subjects were excluded, one due to a neurological abnormality and one due to excessive in-scanner movement. Thus, data from 16 older adults were included in the current analyses.

Demographic and neuropsychological characteristics of the older adults are presented in Table 1. All older adults completed a neuropsychological battery in a separate session no more than 2 years before or after the date of scanning.

2.1.2 Younger adults-Nineteen younger adults participated in this study, but four were excluded due to excessive in-scanner movement and/or task non-compliance. Thus data from 15 younger adults were included in the current analyses. Demographic data for younger adults are also presented in Table 1 . Younger adults did not differ significantly from older adults with respect to years of education $(t=.717, p=.479)$. Note that younger adults did not undergo neuropsychological testing, unlike older adults who completed these as part of a standard laboratory neuropsychological battery.

\subsection{Materials}

2.2.1 Encoding task-The semantic-relatedness encoding task (Mathews, 1977) involves the presentation of triads consisting of a category name and two category exemplars (see Figure 1A). All triads used in this study are identical to those used by Addis and McAndrews (2006), and were constructed using the Battig and Montague (1969) and Murdock (1976) norms, such that only exemplars frequently associated with a category were used.

Over the duration of scanning, 105 encoding triads were shown, 35 of each of three trial types: (1) Triads in which no exemplars relate to the category name ("zero-link" trials); (2) triads in which only one exemplar relates to the category name ("one-link" trials); and (3) triads in which both exemplars relate semantically to the category name ("two-link" trials). Thus, these trials vary in the number of associations provided, from lower-association (zerolink) trials to higher-association (two-link) trials.

In order to counterbalance the use of stimuli in different conditions, categories cycled through the different link conditions. Thus, for each of the 105 category names, 3 triads were constructed (a zero-link, one-link and two-link triad). Moreover, stimuli cycled through runs, so that in each counter-balanced version, category names were presented in a different run. Subjects were randomly assigned to a counterbalanced version. Control trials, consisting of triads of one word corresponding to a response option (i.e., either "none", "one" or "all"; Figure 1B) were interspersed through scanning. Ninety-four baseline trials, consisting of a fixation cross, were also presented through scanning; these ranged in length from 2 to 14 seconds as determined by Optseq2 (Dale, 1999).

2.2.2 Forced-choice recognition task-Identification of successfully encoded triads was based on subsequent recognition of triads during forced-choice recognition. One hundred and five trials, each consisting of an old triad presented during scanning and a new triad (see Figure 1C), were presented. New triads were identical to the old triad, except for one exemplar being replaced with a semantically-related foil which was taken from the same category in the category norms (Battig \& Montague, 1969; Murdock, 1976). The position of the old and new triads (i.e., top or bottom half of the screen) was assigned randomly. Furthermore, the position of the foil (i.e., whether the left or right exemplar is replaced) and whether the foil replaced a related or non-related exemplar in one-link triads was also assigned randomly to triads. 


\subsection{Procedure}

Prior to scanning, participants were familiarized with the encoding task during four practice trials. The instructions emphasized that memory for the encoding triads would be tested after the scan session. Stimuli were presented in black text on a white background and backprojected onto a white screen viewed by the participants through a mirror incorporated into the head coil. E-Prime software (Psychology Software Tools, Inc., Pittsburgh, P.A.) was used for the presentation and timing of stimuli and collection of reaction times and response data. Responses were made on an MR-compatible five-button response box.

The scanning session was divided into 3 runs ( 8 mins $33 s$ ). During each run, $76-80$ trials (baseline, control, zero-, one- and two-link triads) were presented in a pseudo-random order; the order of trial presentation and number and length of baseline trials were determined using Optseq2, an algorithm for optimizing power in event-related fMRI designs (Dale, 1999). Encoding and control triads were presented for $6 s$, considered sufficient for triad encoding (Addis \& McAndrews, 2006; Lepage et al., 2000). For each encoding triad, participants were required to decide how many of the words in the lower portion of the triad could be considered exemplars of the category named in the top portion of the triad. The buttons on the response box assigned to each response were as follows: "none" (right index finger); "one" (right middle finger) or "all" (right ring finger). Thus, the three encoding trial types (zero, one and two link) are identical in terms of the decision task to be performed, and vary only in terms of the number of semantic associations provided. During presentation of control triads (i.e., a triad consisting of either the word "none", "one" or "all"), participants were required to respond according to the word shown (i.e., to select the response key corresponding to "none", "one" or "all").

Immediately following scanning, and approximately 10 minutes after the end of the encoding task, individuals completed the forced-choice recognition task. Each old-new trial was displayed for $6 s$, during which time the participant indicated which triad was seen previously during scanning with a mouse button press. When a response was made (or after $6 s$ if no response was made), the display moved ahead to a fixation cross (presented for $1 s$ ).

\subsection{MR Acquisition and Analysis}

2.4.1 Data acquisition-Images were acquired on a 3 Tesla Siemens Allegra MRI scanner. Detailed anatomical data were collected using a multiplanar rapidly acquired gradient echo (MP-RAGE) sequence. Functional images were acquired using a T2*weighted echo planar imaging (EPI) sequence $(\mathrm{TR}=2000 \mathrm{~ms}, \mathrm{TE}=23 \mathrm{~ms}, \mathrm{FOV}=200 \mathrm{~mm}$, flip angle $=90^{\circ}$ ). Twenty-five coronal oblique slices ( $5 \mathrm{~mm}$ thick) were acquired at an angle parallel to the long axis of the hippocampus in an interleaved fashion.

2.4.2 Data preprocessing-Pre-processing and analyses of imaging data was performed using SPM2 (Wellcome Department of Cognitive Neurology, London, UK). Standard preprocessing of functional images was performed, including discarding the first four functional images to allow scanner equilibrium effects, rigid-body motion correction and unwarping, slice-timing correction, spatial normalization to the Montreal Neurological Institute (MNI) template (resampled at $2 \times 2 \times 2 \mathrm{~mm}$ ) and spatial smoothing (using an $8 \mathrm{~mm}$ full-width half maximum isotropic Gaussian kernel). Data were high-pass filtered to account for low-frequency drifts; a cut-off value of 128 was used.

2.4.3 Parametric modulation analyses-At the fixed effects level, a parametric modulation model was computed for each subject to examine the linear effects of semantic relatedness (i.e., number of semantic links provided) during successful encoding (as determined by subsequent recognition performance); the six head-movement parameters 
were also included as regressors of no interest. Each successfully encoded trial was modeled with a canonical hrf (hemodynamic response function) applied at task onset. Given the possibility that a delayed hrf peak could influence the ability to detect activation in the older adults, we also re-ran the analyses including temporal derivatives of the hrf. This analysis revealed the same pattern of results and is not reported here for brevity ${ }^{1}$. Two contrasts were subsequently specified: one to identify regions in which activity during successful encoding was negatively correlated with the number of associations provided in the stimuli; and another to identify regions in which activity during successful encoding positively correlated with the number of associations. Relevant contrast images were entered into a series of random-effects analyses.

Random-effects conjunction analyses were used to identify those regions in which parametric responses to semantic relatedness during successful encoding were similar across the two age-groups, such that for both groups, neural activity in a region was correlated with the number of associations in either a positive or negative manner. Thus, two conjunction analyses were computed using SPM's masking function to select voxels to include or exclude (e.g., Kensinger and Schacter, 2008). A one-sample $t$-test for one contrast of interest was computed (e.g., positive parametric modulations in younger adults), and activated voxels from this analysis were used to form a mask. A second one-sample $t$-test for the other contrast of interest was computed (e.g., positive parametric modulations in older adults), and the mask from the first analysis was applied, such that the resulting conjunction revealed regions active in both contrasts of interest. Each of the one-sample t-tests created in this process were thresholded at $p<.0225$, resulting in a conjoint voxel-level probability, estimated using Fisher's method (Fisher, 1950; Lazar et al., 2002), of $p<.005$ (note that a cluster-wise approach for correction for multiple comparisons was used; see below).

To identify regions in which parametric responses to semantic relatedness during successful encoding differed across the two age-groups, relevant contrast images were entered into a random-effects independent samples model. In order to account for differences in encoding performance (and the number of successfully encoded trials entering the analysis), we included recognition accuracy as a subject-level covariate. Two contrasts were computed: (1) younger > older adults; and (2) older > younger adults. These contrasts identify voxels for which the slope of the regression line for the covariate of interest (i.e., the number of semantic associations) differs significantly between younger and older adults. This approach can therefore detect voxels in which the slope of the regression line is opposite in sign (e.g., the parametric effect in a region is positive for older adults but negative for younger adults) or of the same sign, but significantly different in magnitude (e.g., the parametric effect is weakly negative for older adults and strongly negative for younger adults). To clarify the nature of any significant differences and to distinguish between these two scenarios (where the slope is of opposite sign or of same sign, but different magnitude), the average estimated slope of the regression line for each group was extracted from relevant beta images to etermine the sign of the effects. Additionally, even if significant group differences emerged, the effects themselves (i.e., for younger or older adults) may not be significantly different from zero. We therefore determined whether effects were significant within group for any regions exhibiting an age-related difference by computing a whole-brain one-sample random-effects $\mathrm{t}$-test for each parametric modulation effect. Thus, four one-sample $t$-tests were computed: negative modulation effects for (1) younger and (2) older adults; and positive modulation effects for (3) younger and (4) older adults. The significance threshold

\footnotetext{
${ }^{1}$ To confirm that there was no delay in the time courses of older adults' neural activity, we also extracted time courses from two regions of interest (left VLPFC and right hippocampus). There was little difference across the age groups in terms of the time at which the hrf peaked. Despite some differences in the height of the hrf across groups and conditions, the hrf peaked between TRs 4 and 5 (8-10 seconds after stimulus onset) in both age-groups.
} 
for these contrast analyses was also set at $p<.005$ uncorrected; note that a cluster-wise approach for correction for multiple comparisons was used (see below).

For all analyses, the minimum cluster size required for corrected significance was determined using a Monte Carlo simulation (10,000 iterations) implemented using AFNI's $3 \mathrm{dClustSim}$ program to estimate the overall probability of false positives within the 3D whole brain search volume $(142,0272 \times 2 \times 2 \mathrm{~mm}$ voxels $)$. Thus, for whole brain results, a combined voxel-wise threshold of $p<.005$ and a spatial extent threshold of 139 voxels was employed to achieve an a of.05, corrected for multiple comparisons. We also computed the required cluster size for the correction of multiple comparisons within our a priori regions of interest (Yassa and Stark, 2008) - the bilateral hippocampus and left VLPFC. Using an anatomical mask comprised of these regions (generated using MARINA; Walter et al., 2003 ) with a search volume of $5,4452 \times 2 \times 2 \mathrm{~mm}$ voxels, the Monte Carlo simulation $(10,000$ iterations) indicated that a voxel-wise threshold of $p<.005$ combined with a spatial extent threshold of 46 voxels was required to correct for multiple comparisons at $p<.05$. For visualization purposes, parameter estimates (beta weights) associated with encoding of zero-, one- and two-link triads were extracted from peak voxels in the left VLPFC and bilateral hippocampus. For localization, peak MNI co-ordinates were converted to Talairach space and localized in reference to a standard stereotaxic atlas (Talairach and Tournoux, 1988).

\section{Results}

\subsection{Behavioral results}

3.1.1 Encoding judgments-The average accuracy and reaction times for the encoding judgements of younger and older adults are presented in Table 2. These responses were collected from all but two participants, for whom data did not record successfully due to a technical failure. A mixed factorial analysis of variance (ANOVA), with a repeated factor of condition (zero-, one- and two-link) and between factor of age-group (young, old) revealed a small effect of condition for accuracy, $F_{2,54}=4.60, p=.01$, and post-hoc polynomial contrasts (linear and quadratic) revealed that this effect reflected a slight linear trend of decreasing accuracy from low (zero-link) to high (two-link) association trials, $F_{1,27}=5.66, p=.03$. The age-groups, however, did not differ in their accuracy during encoding, $F_{1,27}=1.30, p=.26$.

A mixed factorial ANOVA (with repeated factor of condition and between factor of agegroup) of encoding reaction time data also revealed a significant effect of condition, $F_{2,54}=5.06, p=.01$. Post-hoc polynomial contrasts revealed that this effect reflected a quadratic trend in which zero- and two-link triads had longer reaction times than one-link triads, $F_{1,27}=10.65, p=.003$. There was a main effect of age-group, $F_{1,27}=11.94, p=.002$, with younger adults making encoding judgments faster than older adults.

3.1.2 Recognition-Average forced-choice recognition accuracy and reaction time data from all participants are also presented in Table 2. Note that because this was a two-choice recognition task, chance is at $50 \%$, and every participant performed above chance; the lowest performing older adult performed this recognition task with $69 \%$ accuracy. A mixed factorial ANOVA (repeated factor of condition, between factor of age-group) confirmed that there was a significant effect of condition (zero-, one- and two-link) for accuracy, $F_{2,54}=50.69, p<.001$, and a post-hoc polynomial contrast indicated that this effect was due to a linear trend of increasing accuracy from lower (zero-link) to higher (two-link) trials, $F_{1,27}=78.76, p<.001$. There was also a significant effect of age-group, $F_{1,27}=8.16, \mathrm{p}=.008$, with younger adults having higher recognition accuracy than older adults. 
A mixed factorial ANOVA (repeated factor of condition, between factor of age-group) of reaction time data also revealed a significant effect of condition, $F_{2,54}=83.29, p<.001$, and again, this effect reflected a linear decrease in reaction times with increasing associations, $F_{1,27}=127.91, p<.001$. Reaction times also differed significantly between the age-groups, $F_{1,27}=18.44, p<.001$, with younger adults making recognition judgments faster than older adults.

\section{2 fMRI Results}

3.2.1 Negative modulations of neural activity by number of associations-We examined whether neural activity, particularly in left VLPFC (Addis and McAndrews, 2006; Lepage et al., 2000), was negatively correlated with the number of association provided. In other words, were there regions that exhibited more activity during the lower-association trials relative to higher-association trials? A conjunction analysis revealed that there were no regions in which both groups exhibited a common negative modulation of activity. To confirm that this null result did not reflect the two age-groups modulating different subregions of left VLPFC, we conducted a one-sample random-effects $t$-test of negative modulation effects within each group separately. These analyses indicated that in younger adults, left VLPFC activity was significantly modulated by the number of associations, while in older adults, no significant modulation of left VLPFC activity was evident. Moreover, younger adults exhibited negative modulations in a number of regions (right superior temporal gyrus, BA 22, xyz $=57-18-4$; right precuneus, BA 7, $8-4647$; right middle frontal gyrus, BA 6, $32-3$, 54; left inferior parietal lobule, BA 40, -63, -43, 24), while in older adults, no clusters exceeded the significance threshold.

A contrast analysis identified a number of regions in which the negative parametric response to the number of semantic associations (i.e., the slope of the regression line) differed significantly between the age groups (Table 3A). In right superior temporal gyrus (BA 22), inferior (BA 40) and superior parietal (BA 7) lobule, left insula and a region within left VLPFC, specifically, left inferior frontal gyrus (BA 44; Figure 2A), younger adults exhibited a significantly stronger negative modulation effect than older adults, in whom modulations were not significantly different from zero. Examination of the parameter estimates from the activation cluster in left VLPFC suggests that while both age-groups similarly engage left BA 44 during all encoding conditions ${ }^{2}$, younger adults modulated their responses according to the number of semantic associations to be generated while older adults engaged this region in a non-specific manner. There were no regions in which negative modulation effects were significantly stronger in older than younger adults, consistent with the aforementioned observation that older adults did not exhibit any significant negative modulations.

\subsubsection{Positive modulations of neural activity by number of associations-We}

also examined whether neural activity in younger and older adults was positively correlated with the number of association provided, such that more activity was evident during higherversus lower-association trials, when associations are provided for binding. In particular, we were particularly interested in whether hippocampal responses would increase with increasing numbers of associations (Addis and McAndrews, 2006). A conjunction analysis (Table 3B) revealed that in both age groups, positive modulations were evident in left inferior (BA 39/40) and superior (BA 7) parietal lobule, precuneus (BA 19), and middle temporal gyrus (BA 21). Importantly, in both groups right hippocampal activity was significantly modulated in a positive direction by the number of associations (Figure $2 \mathrm{~B}$ ). In

\footnotetext{
${ }^{2}$ A conjunction analysis confirmed that both groups similarly engaged BA 44 during encoding (collapsed across link condition) relative to the control task.
} 
light of this evidence that both groups can modulate hippocampal activity in response to the number of semantic associations, it was surprising that there was not also common modulation of the left hippocampus, given the verbal nature of the task and previous findings of left hippocampal modulation in the same paradigm (Addis \& McAndrews, 2006). To ensure this null result was not simply due to the two age-groups engaging different sub-regions of the left hippocampus during encoding, we conducted a one-sample random-effects t-test of positive modulation effects within each group separately. These analyses revealed that only older adults exhibited a significant positive response to the number of associations in a very anterior region of the left hippocampus $(x y z=-26-5-23$; $k=118$; Figure 2C); no other additional regions emerged from this analysis. Although a positive modulation effect was evident in the left hippocampus of younger adults, the cluster size fell short of the extent threshold required to correct for multiple comparisons ( $x y z=-22$ $-355, k=11$; Figure 2D), and the only other region evident in younger adults was left middle frontal gyrus (BA 10, xyz $=-30,55,5)$. However, contrast analyses did not identify any regions in which the positive parametric responses to the number of semantic associations (i.e., the slope of the regression line) significantly differed between the age groups.

\section{Discussion}

The aim of this study was to differentiate between two mechanisms which could contribute to age-related declines in relational encoding: strategic, generative processes supported by left VLPFC and binding processes supported by the hippocampus. Our findings suggest that left VLPFC dysfunction may underlie relational encoding deficits in older adults. Specifically, unlike younger adults, VLPFC activity in older adults did not vary as a function of the number of semantic associations provided. In contrast, hippocampal activity in both older and younger adults increased with the provision of increasing numbers of associations. Moreover, recognition accuracy improved significantly in both groups with the provision of more semantic associations, indicating the both younger and older adults benefited from this form of encoding support. Overall, these findings suggest that although older adults may be impaired in generating associations, if associations are presented the hippocampus responds to them, binding information that later can be the basis of accurate memory.

One of the key findings of this study was an age-related difference in the up-regulation of left VLPFC (BA 44) according to the generative demands of the encoding task. Although both groups engaged this region across all encoding conditions (relative to the control condition), in younger adults this region was significantly more active during lowerassociation trials that required generation of more associations than higher-association trials. In older adults, this region was active to the same level across all conditions, suggesting that the efficiency of this activation differed with age. This finding is consistent with Sperling and colleagues (Rand-Giovannetti et al., 2006; Sperling et al., 2003) who also report that in older adults, VLPFC activity does not modulate with changes in encoding task demands. In their study, participants were exposed to relational stimuli (name-face pairs) across three presentations. In contrast to younger adults who showed VLPFC activity only during the first presentation of a stimulus, older adults exhibited sustained activation when viewing repeated stimuli. It is possible that the sustained levels of left VLPFC activity in older adults during the higher-association trials did contribute to their increased encoding success, as indexed by increasing subsequent memory performance when more associations were provided. Perhaps when BA 44 is freed up from generating associations, as in the two-link trials, older adults' sustained activity in this region reflects additional processing of the stimuli that contributes to increased later memory for two-link triads. In younger adults, who do not need this additional processing to assist encoding, the activation in this region simply decreases with the generative demands of the task. 
Another key finding is that irrespective of age, right hippocampal engagement was upregulated during encoding when more associations were provided. This finding demonstrates that when older adults have appropriate encoding support - in this case, preexisting associations that linked the to-be-encoded information - the hippocampus is engaged in a manner comparable to younger adults. This positive modulation effect in the hippocampus is also consistent with increased recognition accuracy in higher-association trials, and cannot be attributed to task difficulty (e.g., Leshikar et al., 2010) given that higher-association trials were easier (as indexed by reaction time data). It is important to note that Leshikar et al. (2010) did find the opposite effect - that in both younger and older adults, hippocampal activity was increased during encoding of unrelated versus related words. However, it was not reported whether any regions showed increased activity during the related versus unrelated condition - which may be a possibility, in light of our results.

The current findings further support the view that older adults can, under some conditions, engage the hippocampus to the same extent as younger adults during relational encoding (Duverne et al., 2009 but see Daselaar et al., 2003a, 2003b; Dennis et al., 2008; Sperling, 2007). We posit that one key condition for hippocampal engagement in older adults during relational encoding is the provision of encoding support. When the encoding task is dependent on VLPFC function and the ability to generate semantic associations between the to-be-encoded words - as was the case in the lower-association (zero- and one-link trials) an encoding-related 'deficit' in hippocampal activity may be evident. In contrast, only during higher-association trials did bilateral hippocampal activity increase above baseline in older adults.

There were, however, some age-related differences in the nature of this modulatory hippocampal activity. In younger adults, this effect reflected primarily an increase in hippocampal activity between zero- and one-link triads with highest activity for the one-link trials. However, hippocampal activity in older adults increased across all three conditions and peaked for the two-link trials. Moreover, older adults showed significant modulation of left hippocampus, while this effect was subthreshold in younger adults. It is possible that these differences are related to performance. For example, the younger adults in the current study may have reached some performance ceiling and did not require additional hippocampal activity to successful bind two-link triads that may have become unitized (Quamme et al., 2007).

It is important to note that although the pattern of results in the left VLPFC and hippocampus replicate the findings of previous studies using this paradigm (Addis \& McAndrews, 2006; Lepage et al., 2000), in the current study, activity in these a priori regions of interest did not exceed whole-brain thresholds. Due to the shape and size of the hippocampus, it is often difficult to find clusters that meet whole-brain cluster thresholds unless neighboring regions (such as the parahippocampal gyrus) are also active and the cluster encompasses both regions. Therefore, we took the approach used by Yassa and Stark (2008) for thresholding clusters within the hippocampus, where 3dClustSim takes into account the shape of the structure as well as the number of voxels when computing the extent required for correction. With respect to the VLPFC, it was surprising that clusters did not survive the whole-brain threshold, and while it might be related to sample size, the observation does raise the possibility that only a specific aspect of VLPFC exhibits a modulation effect in response to the task.

With respect to the whole-brain results, it is notable that other prefrontal regions particularly the dorsolateral PFC (DLPFC) - were not evident in the current analyses. Recent work has provided evidence to suggest that the DLPFC may work in conjunction with VLPFC to encode relational information (Blumenfeld et al., 2011). However, other 
studies using this semantic relatedness paradigm also report, when contrasting lower with higher association trials, that prefrontal activity is restricted to VLPFC and does not extend into DLPFC (Addis \& McAndrews, 2006; Lepage et al., 2000), suggesting that VLPFC is particularly engaged when processing sets of unrelated items and demands on generative processes is high. In contrast, DLPFC may play a more general role in all forms of relational encoding irrespective of the pre-existing semantic relations and is emergent when contrasted against unsuccessful encoding or item encoding, and thus could not be isolated in the absence of these comparison conditions.

Whole-brain results revealed evidence of neural commonalities and differences across agegroups outside of the left VLPFC and hippocampus. For instance, in both age groups, neural activity in left lateral temporal and parietal cortex was up-regulated when more associations were presented. Addis and McAndrews (2006) speculated that lateral temporal responses may reflect access to the semantic knowledge on which the provided associations are based, while the lateral parietal cortex may provide an interface between lateral and medial temporal regions. Another possibility is that lateral temporal activity reflects gist-based processing which is heightened when all presented words are semantically associated (Dennis et al., 2008b), while lateral parietal activity reflects the maintenance of the to-beencoded verbal information in working memory (e.g., Gold and Buckner, 2002). In contrast, for lower-association trials, age differences emerged in a number of regions including right posterior superior temporal gyrus and right lateral parietal cortex (BA 40). Previous work has demonstrated these regions to be active during the encoding of lower-association trials in younger adults (Addis and McAndrews, 2006), and more so than older adults particularly during lower-association trials (Leshikar et al., 2010). In general, this pattern of results is similar to that reported for the left VLPFC and hippocampus: Neural differences emerge when no encoding support is provided, but when more associations between to-be-encoded information are provided, older adults can engage many of the same regions as younger adults. This finding further supports the idea that the strategic organization of information may be particularly sensitive to disruption in aging, but may be ameliorated by the use of specific encoding strategies (see also Leshikar et al., 2010 for a similar view).

One limitation of the current paradigm is that the encoding task did not involve an explicit instruction to "generate" a relationship between semantic associates. However, it is highly likely that the evaluative process required to assess the number of semantic relationships evident in each triad would entail some level of semantic elaboration. Importantly, the process of semantic elaboration is associated with activation of the left VLPFC (Han et al., 2012; Raposo et al., 2009), and may be particularly evident when semantic elaboration can enhance memory performance (Raposo et al., 2009). Thus, the up-regulation of left VLPFC when generative demands are higher - in this and other studies (Addis and McAndrews, 2006; Lepage et al., 2000; see also, Leshikar et al., 2010) - is consistent with increased semantic elaboration processes during successful encoding. Indeed, the use of semantic elaboration strategies engages left VLPFC during encoding (Kirchhoff and Buckner, 2006) and given that our participants completed this encoding task knowing that a memory test would follow the fMRI session, it is highly likely that the instruction to focus on semantic associations provided an encoding strategy that participants could utilize.

It is also interesting to note that during the encoding task, participants in both age groups were slightly (but significantly) less accurate at judging the number of associations present in the two-link trials. Combined with the fact that participants were also faster on these trials, it is possible that individuals made a speed-accuracy trade-off. Unlike one-link triads, two-link triads require evaluation of the entire triad for a correct response to be made and thus individuals may sometimes respond after recognizing one association before noticing a second. 


\section{Conclusions}

In summary, the current findings suggest that left VLPFC dysfunction may underlie agerelated deficits in relational encoding by disrupting the strategic organization to-be-encoded information by generating associations. However, when associations - such as pre-existing semantic associations between items - are available, hippocampal activity in older adults is up-regulated to the level of younger adults, and encoding performance is enhanced. These findings underscore the notion that when examining age-related changes in encoding, a finegrained analysis considering the generative demands and encoding support provided by the encoding task, is of critical importance to understanding the similarities and differences between younger and older adults.

\section{Acknowledgments}

This research was funded by a grant from the National Institute on Aging to D.L.S. D.R.A. was supported by Rutherford Discovery Fellowship RDF-10-UOA-024, and K.S.G. was supported by NIA grant AG028774. We thank Nicole Anderson for helpful input on experimental design.

\section{References}

Achim AM, Lepage M. Neural correlates of memory for items and associations: an event-related functional magnetic resonance imaging study. Journal of cognitive neuroscience. 2005; 17:652-667. [PubMed: 15829085]

Addis DR, McAndrews MP. Prefrontal and hippocampal contributions to the generation and binding of semantic associations during successful encoding. NeuroImage. 2006; 33:1194-1206. [PubMed: 17023179]

Anderson, ND.; Craik, FIM. Memory in the aging brain. In: Tulving, E.; Craik, FIM., editors. The Oxford Handbook of Memory. Oxford University Press; New York N.Y.: 2000. p. 411-425.

Battig WF, Montague WE. Category norms for verbal items in 56 categories: a replication and extension of the Connecticut Category Norms. Experimental Psychology Monographs. 1969; 80:146.

Blumenfeld RS, Parks CM, Yonelinas AP, Ranganath C. Putting the pieces together: The role of dorsolateral prefrontal cortex in relational memory encoding. Journal of cognitive neuroscience. 2011; 23:257-265. [PubMed: 20146616]

Buckner RL. Functional-anatomic correlates of control processes in memory. The Journal of Neuroscience. 2003; 15:3999-4004. [PubMed: 12764084]

Bunce D. Cognitive support at encoding attenuates age differences in recollective experience among adults of lower frontal lobe function. Neuropsychology. 2003; 17:353-361. [PubMed: 12959501]

Burke DM, Light LL. Memory and aging: The role of retrieval processes. Psychological review. 1981; 90:513-546.

Cabeza R, Grady C, Nyberg L, McIntosh AR, Tulving E, Kapur S, Jennings JM, Houle S, Craik FIM. Age-related differences in neural activity during memory encoding and retrieval: A positron emission tomography study. The Journal of Neuroscience. 1997; 17:391-400. [PubMed: 8987764]

Cabeza R, Nyberg L. Imaging cognition II: An empirical review of 275 PET and fMRI studies. Journal of cognitive neuroscience. 2000; 12:1-47. [PubMed: 10769304]

Chalfonte BL, Johnson MK. Feature memory and binding in young and older adults. Memory and cognition. 1996; 24:403-416. [PubMed: 8757490]

Chua E, Schacter DL, Rand-Giovannetti E, Sperling RA. Evidence for a specific role of the anterior hippocampal region in successful associative encoding. Hippocampus. 2007; 17:1071-1080. [PubMed: 17604351]

Craik, FIM. A functional account of age differences in memory. In: Klix, F.; Hagendorf, H., editors. Human Memory and Cognitive Capabilities, Mechanisms and Performances. Elsevier; Amsterdam: 1986. p. 409-422. 
Dale AM. Optimal experimental design for event-related fMRI. Human brain mapping. 1999; 8:109_ 114. [PubMed: 10524601]

Daselaar SM, Veltman DJ, Rombouts SA, Raaijmakers JG, Jonker C. Deep processing activates the medial temporal lobe in young but not in old adults. Neurobiology of aging. 2003a; 24:1005-1011. [PubMed: 12928060]

Daselaar SM, Veltman DJ, Rombouts SA, Raaijmakers JG, Jonker C. Neuroanatomical correlates of episodic encoding and retrieval in young and elderly subjects. Brain: a journal of neurology. 2003b; 126:43-56. [PubMed: 12477696]

Davachi L, Mitchell JP, Wagner AD. Multiple routes to memory: distinct medial temporal lobe processes build item and source memories. Proceedings of the National Academy of Sciences of the United States of America. 2003; 100:2157-2162. [PubMed: 12578977]

Davis HP, Klebe KJ, Guinther PM, Schroder KB, Cornwell RE, James LE. Subjective organization, verbal learning, and forgetting across the life span: from 5 to 89. Experimental aging research. 2013; 39:1-26. [PubMed: 23316734]

Dennis NA, Daselaar S, Cabeza R. Effects of aging on transient and sustained successful memory encoding activity. Neurobiology of aging. 2007; 28:1749-1758. [PubMed: 16919850]

Dennis NA, Hayes SM, Prince SE, Madden DJ, Huettel SA, Cabeza R. Effects of aging on the neural correlations of successful item and source memory encoding. Journal of Experimental Psychology: Learning, Memory, \& Cognition. 2008a; 34:791-808.

Dennis NA, Kim H, Cabeza R. Age-related differences in brain activity during true and false memory retrieval. Journal of cognitive neuroscience. 2008b; 20:1390-1402. [PubMed: 18303982]

Duverne S, Motamedinia S, Rugg MD. The relationship between aging, performance, and the neural correlates of successful memory encoding. Cerebral cortex. 2009; 19:733-744. [PubMed: 18653664]

Eichenbaum H, Yonelinas AP, Ranganath C. The medial temporal lobe and recognition memory. Annual Review of Neuroscience. 2007; 30:123-152.

Fernandez G, Tendolkar I. Integrated brain activity in medial temporal and prefrontal areas predicts subsequent memory performance: human declarative memory formation at the system level. Brain research bulletin. 2001; 55:1-9. [PubMed: 11427332]

Fisher, RA. Statistical Methods for Research Workers. Oliver \& Boyd; London: 1950.

Fletcher P, Shallice T, Dolan RJ. "Sculpting the response space" -- an account of left prefrontal activation at encoding. NeuroImage. 2000; 12:404-417. [PubMed: 10988034]

Gold BT, Buckner RL. Common prefrontal regions coactivate with dissociable posterior regions during controlled semantic and phonological tasks. Neuron. 2002; 35:803-812. [PubMed: 12194878]

Grady CL, McIntosh AR, Horwitz B, Maisog JM, Ungerleider LG, Mentis MJ, Schapiro MB, Haxby JV. Age-related reductions in human recognition memory due to impaired encoding. Science. 1995; 269:218-221. [PubMed: 7618082]

Han S, O'Connor AR, Eslick AN, Dobbins IG. The role of left ventrolateral prefrontal cortex during episodic decisions: semantic elaboration or resolution of episodic interference? Journal of cognitive neuroscience. 2012; 24:223-234. [PubMed: 21916561]

Henke K, Weber B, Kneifel S, Wieser HG, Buck A. Human hippocampus associates information in memory. Proceedings of the National Academy of Sciences of the United States of America. 1999; 96:5884-5889. [PubMed: 10318979]

Jackson O 3rd, Schacter DL. Encoding activity in anterior medial temporal lobe supports subsequent associative recognition. NeuroImage. 2004; 21:456-462. [PubMed: 14741683]

Jennings JM, Jacoby LL. Automatic versus intentional uses of memory: aging, attention, and control. Psychology and aging. 1993; 8:283-293. [PubMed: 8323731]

Kensinger EA, Schacter DL. Neural processes supporting young and older adults' emotional memories. Journal of cognitive neuroscience. 2008; 20:1161-1173. [PubMed: 18284340]

Kirchhoff BA, Anderson BA, Barch DM, Jacoby LL. Cognitive and neural effects of semantic encoding strategy training in older adults. Cerebral cortex. 2012; 22:788-799. [PubMed: 21709173] 
Kirchhoff BA, Buckner RL. Functional-anatomic correlates of individual differences in memory. Neuron. 2006; 51:263-274. [PubMed: 16846860]

Kirwan CB, Stark CE. Medial temporal lobe activation during encoding and retrieval of novel facename pairs. Hippocampus. 2004; 14:919-930. [PubMed: 15382260]

Lazar NA, Luna B, Sweeney JA, Eddy WF. Combining brains: a survey of methods for statistical pooling of information. NeuroImage. 2002; 16:538-550. [PubMed: 12030836]

Lepage M, Habib R, Cormier H, Houle S, McIntosh AR. Neural correlates of semantic associative encoding in episodic memory. Brain Research: Cognitive Brain Research. 2000; 9:271-280. [PubMed: 10808138]

Leshikar ED, Gutchess AH, Hebrank AC, Sutton BP, Park DC. The impact of increased relational encoding demands on frontal and hippocampal function in older adults. Cortex; a journal devoted to the study of the nervous system and behavior. 2010; 46:507-521.

Light, LL.; Prull, MW.; LaVoie, DJ.; Healy, MR. Dual-process theories of memory in old age. In: Perfect, TJ.; Maylor, EA., editors. Models of Cognitive Aging. Oxford University Press; New York N.Y.: 2000. p. 238-300.

Logan JM, Sanders AL, Snyder AZ, Morris JC, Buckner RL. Under-recruitment and nonselective recruitment: dissociable neural mechanisms associated with aging. Neuron. 2002; 33:827-840. [PubMed: 11879658]

Lyle KB, Bloise SM, Johnson MK. Age-related binding deficits and the content of false memories. Psychology and aging. 2006; 21:86-95. [PubMed: 16594794]

Mathews RC. Semantic judgments as encoding operations: the effects of attention to particular semantic categories on the usefulness of interitem relations in recall. Journal of Experimental Psychology: Human Learning and Memory. 1977; 3:160-173.

McGillivray S, Castel AD. Memory for age-face associations in younger and older adults: the role of generation and schematic support. Psychology and aging. 2010; 25:822-832. [PubMed: 21058867]

Mitchell KJ, Johnson MK, Raye CL, Mather M, D'Esposito M. Aging and reflective processes of working memory: binding and test load deficits. Psychology and aging. 2000; 15:527-541. [PubMed: 11014715]

Morcom AM, Good CD, Frackowiak RS, Rugg MD. Age effects on the neural correlates of successful memory encoding. Brain: a journal of neurology. 2003; 126:213-229. [PubMed: 12477708]

Moscovitch M. Memory and working-with-memory: a component process model based on modules and central systems. Journal of cognitive neuroscience. 1992; 4:257-267. [PubMed: 23964882]

Moscovitch M, Winocur G. Frontal lobes, memory, and aging. Annals of the New York Academy of Sciences. 1995; 769:119-150. [PubMed: 8595020]

Mottaghy FM, Shah NJ, Krause BJ, Schmidt D, Halsband U, Jäncke L, Müller-Gärtner HW. Neuronal correlates of encoding and retrieval in episodic memory during a paired-word association learning task: a functional magnetic resonance imaging study. Experimental Brain Research. 1999; 128:332-342.

Murdock BB. Item and order information in short-term serial memory. Journal of experimental psychology: General. 1976; 105:191-216.

Murray LJ, Ranganath C. The dorsolateral prefrontal cortex contributes to successful relational memory encoding. Journal of Neuroscience. 2007; 27:5515-5522. [PubMed: 17507573]

Naveh-Benjamin M. Adult age differences in memory performance: Tests of an associative deficit hypothesis. Journal of Experimental Psychology: Learning, Memory, \& Cognition. 2000; 26:1170-1187.

Old SR, Naveh-Benjamin M. Differential effects of age on item and associative measures of memory: a meta-analysis. Psychology and Aging. 2008; 23:104-118. [PubMed: 18361660]

Prince SE, Daselaar SM, Cabeza R. Neural correlates of relational memory: successful encoding and retrieval of semantic and perceptual associations. The Journal of neuroscience: the official journal of the Society for Neuroscience. 2005; 25:1203-1210. [PubMed: 15689557]

Quamme JR, Yonelinas AP, Norman KA. Effect of unitization on associative recognition in amnesia. Hippocampus. 2007; 17:192-200. [PubMed: 17203466] 
Rand-Giovannetti E, Chua EF, Driscoll AE, Schacter DL, Albert MS, Sperling RA. Hippocampal and neocortical activation during repetitive encoding in older persons. Neurobiology of aging. 2006; 27:173-182. [PubMed: 16298252]

Raposo A, Han S, Dobbins IG. Ventrolateral prefrontal cortex and self-initiated semantic elaboration during memory retrieval. Neuropsychologia. 2009; 47:2261-2271. [PubMed: 19038275]

Ryan JD, Leung G, Turk-Browne NB, Hasher L. Assessment of age-related changes in inhibition and binding using eye movement monitoring. Psychology and aging. 2007; 22:239-250. [PubMed: 17563180]

Spaniol J, Davidson PS, Kim AS, Han H, Moscovitch M, Grady CL. Event-related fMRI studies of episodic encoding and retrieval: meta-analyses using activation likelihood estimation. Neuropsychologia. 2009; 47:1765-1779. [PubMed: 19428409]

Sperling RA. Functional MRI studies of associative encoding in normal aging, mild cognitive impairment, and Alzheimer's disease. Annals of the New York Academy of Sciences. 2007; 1097:146-155. [PubMed: 17413017]

Sperling RA, Bates JF, Chua EF, Cocchiarella AJ, Rentz DM, Rosen BR, Schacter DL, Albert MS. fMRI studies of associative encoding in young and elderly controls and mild Alzheimer's disease. Journal of Neurology, Neurosurgery \& Psychiatry. 2003; 74:44-50.

Staresina BP, Davachi L. Selective and shared contributions of the hippocampus and perirhinal cortex to episodic item and associative encoding. Journal of Cognitive Neuroscience. 2008; 20:14781489. [PubMed: 18303974]

Staresina BP, Davachi L. Mind the gap: binding experiences across space and time in the human hippocampus. Neuron. 2009; 63:267-276. [PubMed: 19640484]

Talairach, J.; Tournoux, P. Co-Planar Stereotaxic Atlas of the Human Brain. Thieme Medical Publishers, Inc; New York, N.Y.: 1988.

Walter, B.; Blecker, C.; Kirsch, P.; Sammer, G.; Schienle, A.; Stark, R.; Vaitl, D. MARINA: An easy to use tool for the creation of MAsks for Region of INterest Analyses; 9th International Conference on Functional Mapping of the Human Brain; New York, NY. 2003.

Yassa MA, Stark CE. Multiple signals of recognition memory in the medial temporal lobe. Hippocampus. 2008; 18:945-954. [PubMed: 18493928] 


\section{(A) Encoding (scanned)}

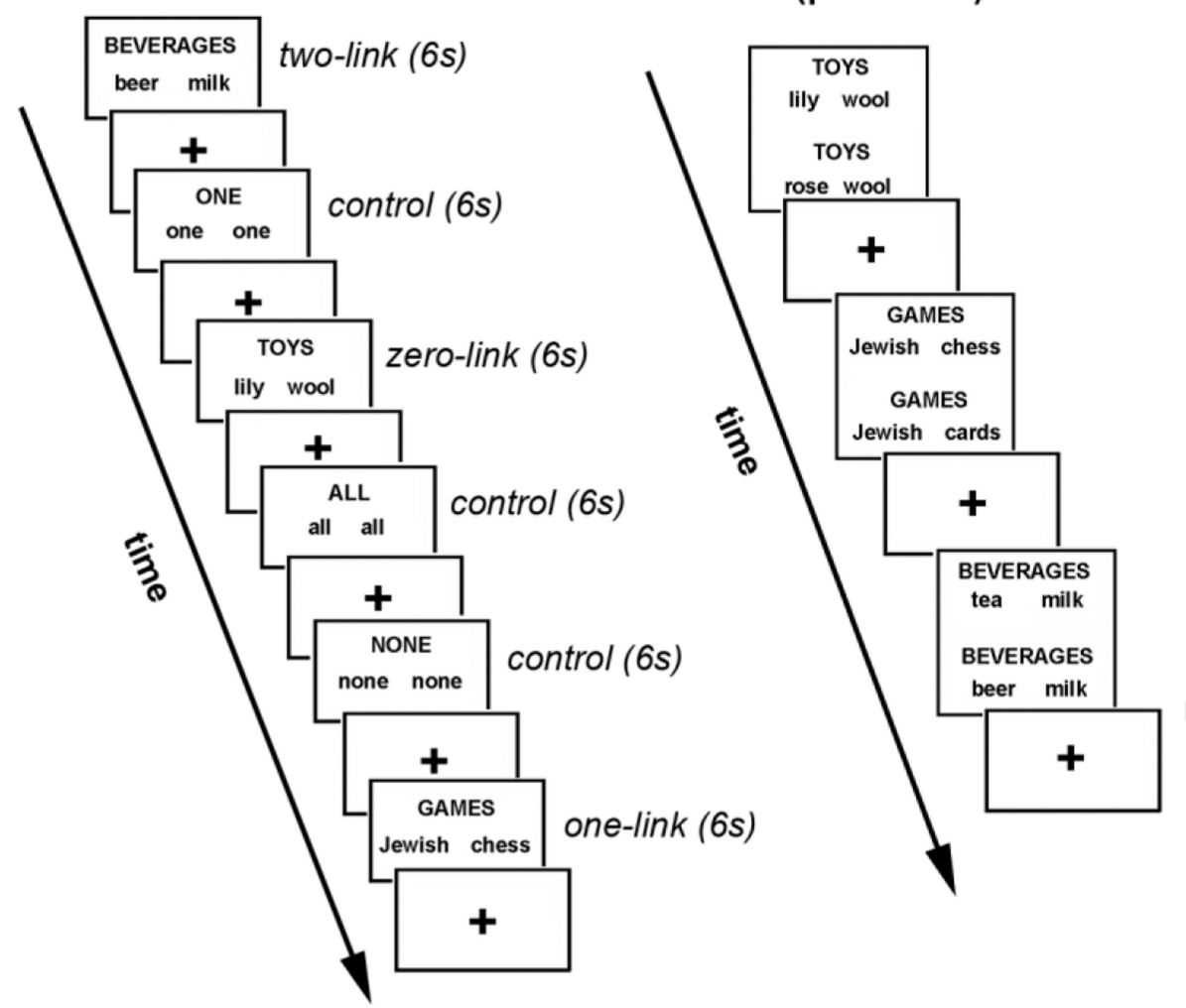

Figure 1.

Experimental paradigm. (A) Examples of to-be-encoded triads from the zero-, one- and twolink conditions. Each triad consists of a category name (top) and two category exemplars (bottom) which are either semantically related or unrelated to the category, depending on the condition. Participants identified how many exemplars (none, one or all) fit in the category with a button press. Control trials are also shown where participants were required to press the button corresponding to the response shown. All trials were separated by presentation of a fixation cross (of jittered duration; see Methods). (B) Examples of forced choice recognition trials, in which one previously seen triad and one new triad (containing one foil exemplar), presented during a post-scan recognition test. Participants identified which of the two triads were presented during encoding. 

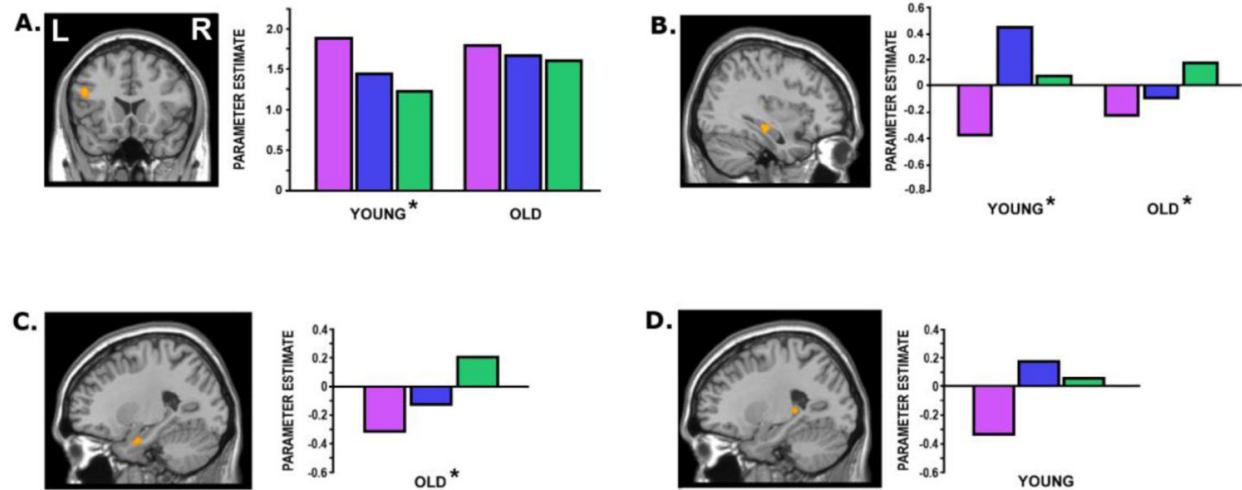

ZERO-LINK

ONE-LINK

TWO-LINK

Figure 2.

VLPFC and hippocampal regions in which activity was modulated by to the number of associations provided during encoding. Younger adults exhibited a significantly stronger negative response relative to older adults in BA 44 (A: $x y z=-4820$ 26). In the right hippocampus, both age-groups exhibited a common positive response (B: $x y z=36-26-9$ ). In older adults, left anterior hippocampal activity was also modulated by the number of associations (C: $x y z=-26-5-23)$, but a modulation effect in the left posterior hippocampus was subthreshold in younger adults (D: $x y z=-22-355$ ). Activations shown at $p=.005$ uncorrected, overlaid on a standard template. For all regions, mean parameter estimates (beta weights) associated with the successful encoding of zero-, one- and two-link triads are plotted for illustrative purposes only (and thus error bars are not shown). *Significant modulation effects, as indicated by one-sample random-effects analyses. L=left; $\mathrm{R}=$ right. 
Table 1

Demographic and neuropsychological characteristics of younger and older participants

\begin{tabular}{lcc}
\hline & \multicolumn{2}{c}{ Group means (sd): } \\
\cline { 2 - 3 } Demographic/neuropsychological characteristic & Younger & Older \\
\hline Sex & $7 \mathrm{M} / 8 \mathrm{~F}$ & $6 \mathrm{M} / 10 \mathrm{~F}$ \\
Age (in years) & $24.00(3.05)$ & $72.63(5.55)$ \\
Education (in years) & $16.07(2.46)$ & $15.44(2.42)$ \\
CVLT (cued delayed recall; maximum 16) & n/a & $13.56(2.16)$ \\
Digit Span Backwards (maximum 14) ${ }^{\dagger}$ & $\mathrm{n} / \mathrm{a}$ & $8.33(2.53)$ \\
Mini-Mental State Examination (maximum 30) & $\mathrm{n} / \mathrm{a}$ & $29.50(0.97)$ \\
Phonemic (FAS) fluency (total score; no maximum) & $\mathrm{n} / \mathrm{a}$ & $49.00(12.59)$ \\
Verbal Paired Associates I (Recall Total Score; maximum 32) & $\mathrm{n} / \mathrm{a}$ & $22.38(5.19)$ \\
Wisconsin Card Sort Test (number of categories; maximum 6) & n/a & $5.25(1.61)$ \\
\hline
\end{tabular}

Note. CVLT $=$ California Verbal Learning Test.

${ }^{\dagger}$ One subject did not complete Digit Span Backwards and thus the mean/standard deviation reported is based on 15 subjects. 


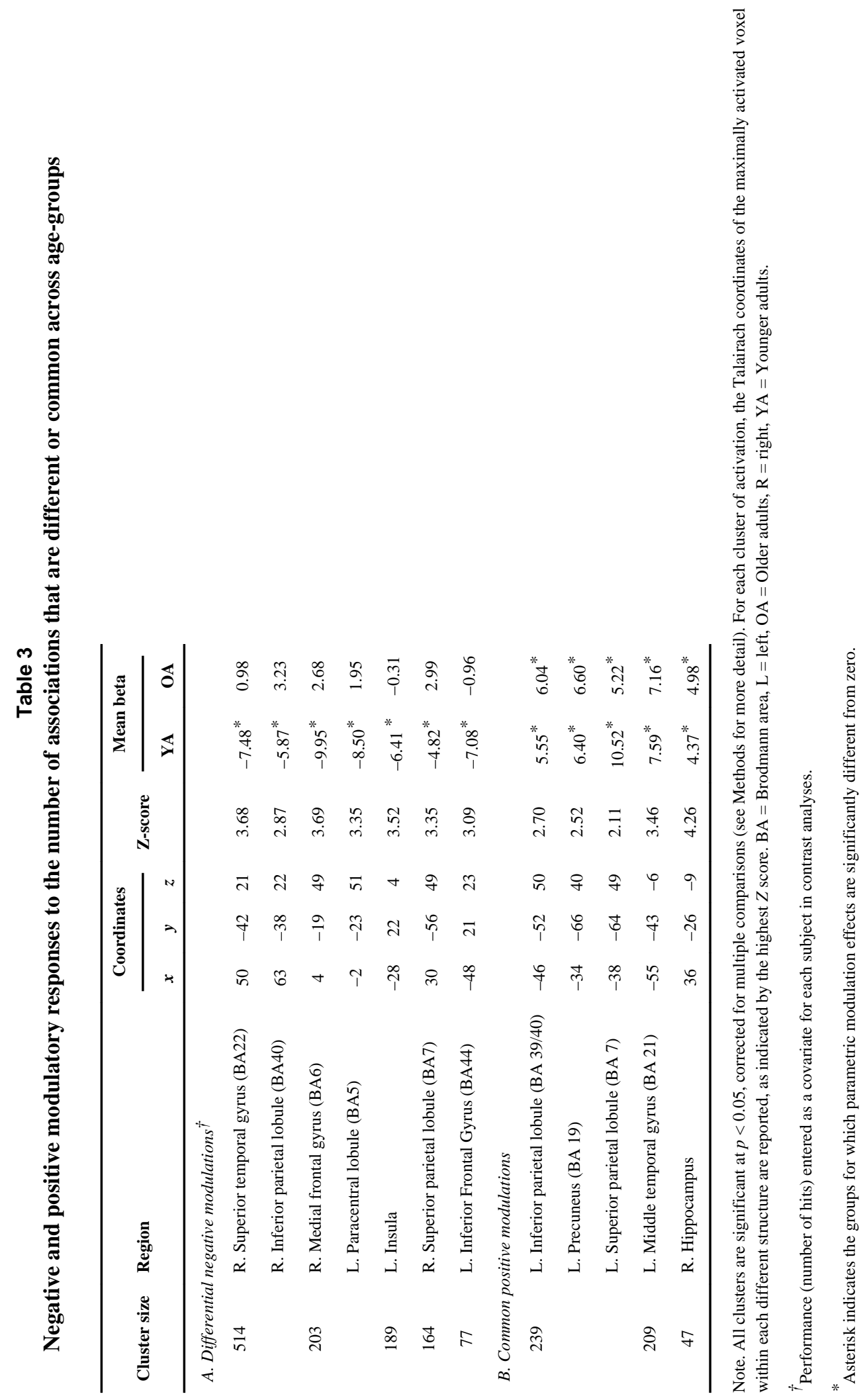

